\title{
An Overview of Metabolic Phenotyping in Blood Pressure Research
}

\author{
Ioanna Tzoulaki ${ }^{1,2,3} \cdot$ Aikaterini Iliou $^{4} \cdot$ Emmanuel Mikros $^{4} \cdot$ Paul Elliott $^{1,2,5,6}$
}

Published online: 10 July 2018

(C) The Author(s) 2018

\begin{abstract}
Purpose of the Review This review presents the analytical techniques, processing and analytical steps used in metabolomics phenotyping studies, as well as the main results from epidemiological studies on the associations between metabolites and high blood pressure.

Recent Findings A variety of metabolomic approaches have been applied to a range of epidemiological studies to uncover the pathophysiology of high blood pressure. Several pathways have been suggested in relation to blood pressure including the possible role of the gut microflora, inflammatory, oxidative stress, and lipid pathways. Metabolic changes have also been identified associated with blood pressure lowering effects of diets high in fruits and vegetables and low in meat intake. However, the current body of literature on metabolic profiling and blood pressure is still in its infancy, not fully consistent and requires careful interpretation.

Summary Metabolic phenotyping is a promising approach to uncover metabolic pathways associated with high blood pressure and throw light into the complex pathophysiology of hypertension.
\end{abstract}

Keywords Blood pressure $\cdot$ Hypertension $\cdot$ Metabolomics $\cdot$ Metabolic phenotyping $\cdot$ Microbiome $\cdot$ Epidemiological studies

\section{Introduction}

High blood pressure is the leading modifiable cause of death worldwide; even small increments in blood pressure are associated with an increased risk of cardiovascular disease (CVD) $[1 \bullet 2]$. Extensive research has confirmed independent

This article is part of the Topical Collection on Prevention of Hypertension: Public Health Challenges

Ioanna Tzoulaki

i.tzoulaki@imperial.ac.uk

1 Department of Epidemiology and Biostatistics, School of Public Health, Imperial College London, London, UK

2 MRC-PHE Centre for Environment and Health, School of Public Health, Imperial College London, London, UK

3 Department of Hygiene and Epidemiology, University of Ioannina Medical School, Ioannina, Greece

4 Department of Pharmaceutical Chemistry, Faculty of Pharmacy, University of Athens, Athens, Greece

5 Health Data Research UK (HDR-UK), London, UK

6 Dementia Research Institute at Imperial College London, London, UK unfavorable additive effects on blood pressure of adiposity (body mass), excess alcohol use, high salt intake, and inadequate potassium intake, as well as beneficial effects from use of the Diet Approaches to Stop Hypertension (DASH) feeding trial combination diet [3-8]. Yet the effect of other nutrients, environmental factors as well as the mechanisms through which known dietary factors affect blood pressure are less well understood.

Metabolic phenotyping (metabolomics), the study of low molecular weight molecules or metabolites produced within cells and biologic systems, offers a promising approach to measure biological effects of environmental and other exposures and discover novel disease biomarkers [9••] with applications to blood pressure research. Here, we outline the main analytical approaches in metabolic phenotyping research and then discuss its application to blood pressure research in human studies, focusing on recent epidemiological studies investigating metabolic markers and pathways associated with blood pressure.

\section{Metabolic Phenotyping}

High-throughput metabolic phenotyping or profiling is a powerful tool in systems biology [10] that is being increasingly 
applied to biomarker discovery. It refers to the biochemical quantitative analysis of multiple metabolites in biological fluids, tissues, and tissue extracts. Specifically, global (untargeted) metabolic profiling provides a comprehensive assessment of the metabolic effects of intrinsic markers and extrinsic exposures (the internal and external exposome) from a variety of sources including, but not limited to, dietary, lifestyle, gut microbial, and psychosocial factors [9••, 11, 12•]. These factors interact at a cellular and systems level to generate a metabolic signature characteristic of health or disease [13].

Low molecular weight metabolites are intermediates or end products of cellular processes and as an ensemble characterise the function of an organism. The main analytical methods used are proton Nuclear Magnetic Resonance ( ${ }^{1} \mathrm{H}$ NMR) spectroscopy, and Mass Spectrometry (MS) coupled to an array of separation techniques including Gas Chromatography (GC) and Liquid Chromatography (LC). These methods are complementary to each other [14] with different strengths and weaknesses (Fig. 1) and allow the simultaneous detection and quantitation of a variety of low-molecular-weight metabolites including carbohydrates, lipids, organic acids, amino acids, energy-related metabolites and gut microbial co-metabolites, with concentrations ranging from picomoles per litre to millimoles per litre. The wealth of resulting spectral data can be analysed using emerging methods for automated data reduction and pattern recognition techniques (multivariate chemometric analysis) leading to the efficient exploitation of complex spectral profiles and extraction of latent information [15-18].

${ }^{1} \mathrm{H}$ NMR spectroscopy is based on the excitation properties of protons in the presence of magnetic field. NMR-active nucleus absorbs electromagnetic radiation at characteristic frequencies providing information about the structure and abundance of the molecule, enabling both identification and quantitation. NMR is a non-destructive method characterised by high reproducibility [19-21]. Different NMR techniques can be used for particular type of analysis like magic angle spinning (MAS)-NMR to study intact tissues or lipoprotein profiling for lipoprotein particle number and size, and lipoprotein subfractions in blood samples by scrutinising the shape of the signal envelopes [22]. NMR is restricted by means of sensitivity and the number of metabolites that can be detected by a single run. High abundance metabolites may cause masking of lower abundance molecules. To overcome the latter, prior sample preparation $[23,24]$ (e.g. separation between aqueous and lipid phase), two-dimensional NMR spectra [25], or hyphenation of NMR with separation techniques [26] can be used.

By contrast, MS is characterised by high sensitivity ( $1 \mathrm{pmol} / \mathrm{L})$, allowing the detection of less abundant metabolites. It is particularly useful for global metabolite profiling, as thousands of metabolites can be detected in a single run [27, 28]. Its principle is that molecules are charged or ionised and then charged molecules and their fragments are separated
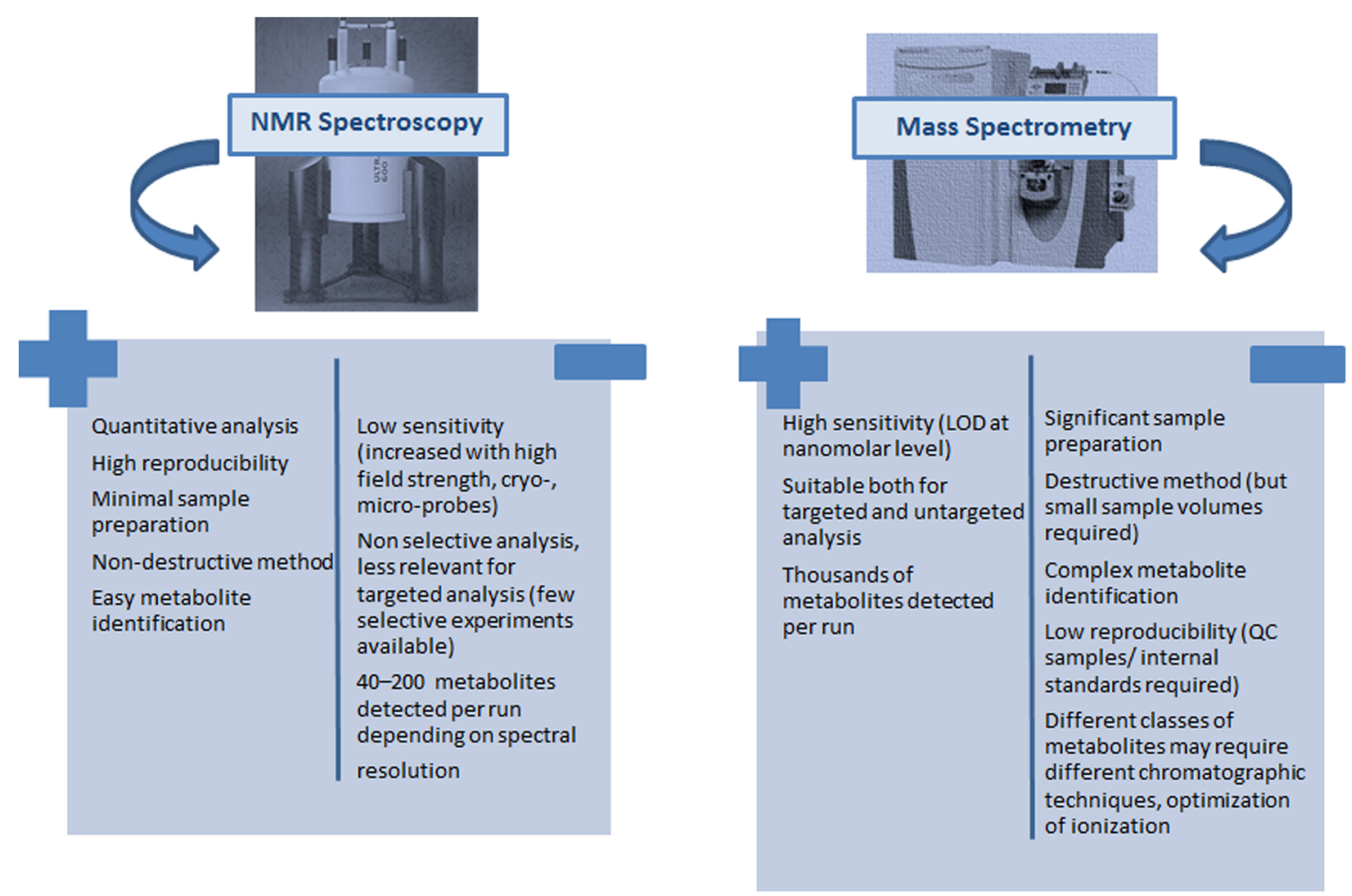

Fig. 1 Strengths and weaknesses of NMR Spectroscopy and MS spectrometry as an analytical technique in metabolomics research 
based on the $\mathrm{m} / \mathrm{z}$ ratio (mass-to-charge ratio). In metabolic phenotyping, where small molecules are studied, $z$ is equal to one, so that $\mathrm{m} / \mathrm{z}$ ratio coincides with the mass. Different ionisation techniques can be used, with Electrospray Ionization Technique (ESI), both in negative and positive mode, being the most applicable in metabolic phenotyping research $[29,30]$. Major disadvantages of MS technique include that it is a destructive method, though a smaller sample is needed compared to NMR. Attention should be paid to sample preparation, as hydrophobicity and ionisation potential affects the analysis. When hyphenated with GC, a derivatisation process is needed [31 •]. Moreover, MS is characterised by low reproducibility, necessitating the use of internal standards and quality control samples [32]. The latter may be pooled samples, consisting of a small amount of each one of the study samples, predetermined mixtures of "representative" metabolites or samples commercially available.

Metabolic phenotyping through NMR or MS can be hypothesis driven, named as a targeted approach [33], where only a preselected group of metabolites is measured, usually related to the pathway of interest or a specific class of metabolites. In targeted methods, chemical identification (annotation) of the metabolites is completed at the development phase and also serves for validation of potential biomarkers previously discovered. On the other hand, untargeted approaches are hypothesis generating and allow for unbiased detection of a wide range of metabolites and therefore may help reveal novel metabolic pathway or biomarkers. However, structural identification of the metabolic features can be complex and often annotation of the metabolic features that are of interest is incomplete [27, 34, 35].

The most frequently used samples in human metabolomics studies are blood and urine as they are relatively easy to collect and provide a comprehensive overview of various system level metabolic pathways [36, 37]. Metabolic phenotyping can also be undertaken of other biological fluids (e.g. saliva), tissue extracts from biopsies, or cell extracts [38-41]. These samples may reflect pathophysiological changes in specific tissues or cells that may not be captured by blood and urine.

Data analysis of metabolic phenotyping data is relatively complex (Fig. 2). It may comprise, among others, initial steps of processing to correct for instrumental drift [42, 43], methods for peak alignment/integration [44-46], correction of sample dilution effects [47], quality control of variables, and later use of multi- or univariate statistical methods adjusting for covariates, in order to find associations with health outcomes. The complexity of the task increases in studies with subsets of data acquired by different platforms, instruments, and methods $[31 \bullet, 48 \cdot]$. In untargeted analyses in particular, attention needs to be paid to the high dimensionality of the data, the large inter-correlations between data points, and the problems of multiple testing $[31 \bullet, 49]$.

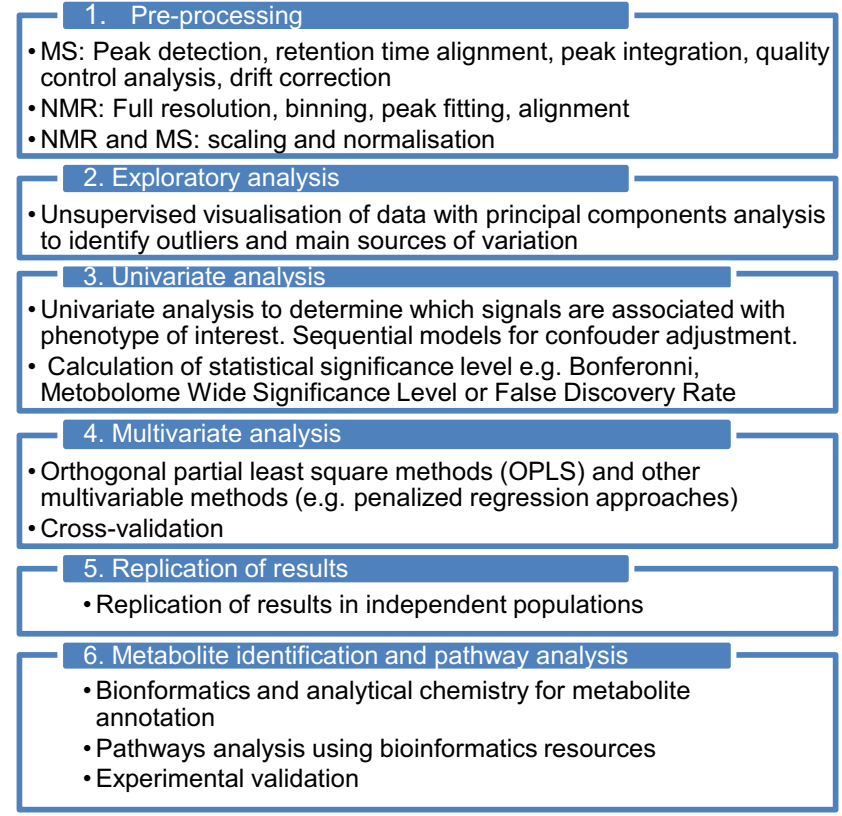

Fig. 2 Data analysis workflow for metabolic phenotyping studies

\section{Epidemiological Studies on Metabolic Phenotyping in Blood Pressure Research}

Applications of metabolic phenotyping to blood pressure research have been multifaceted, including the study of lifestyle and environmental factors on blood pressure levels through biomarkers of exposure [50-61], investigation of disease pathophysiological processes [62-70] and evaluation of drug response to treatment for high blood pressure and associated side effects [71-77]. However, despite increasing interest in this area, most studies are still small, cross-sectional, and limited to targeted metabolic platforms.

By far, the largest piece of evidence regarding small metabolites and pathogenesis of high blood pressure comes from the INTERnational collaborative study on Macro/ micronutrients And blood Pressure (INTERMAP) that included 4630 men and women aged 40-59 years from 17 population samples in the USA, UK, Peoples' Republic of China, and Japan [55••]. The study has a unique collection of four interviewer-administered multi-pass 24-h dietary recalls allowing for a comprehensive assessment of dietary habits, and two 24-h urine collections from each individual. ${ }^{1} \mathrm{H}$ NMR-based untargeted metabolic phenotyping of urine was performed on the stored 24-h urine collections from the 4630 participants. These analyses highlighted discriminatory metabolites across the four countries, of which four metabolites (alanine, hippurate, formate, and $N$-methylnicotinate) were associated with blood pressure of individuals. Two of these metabolites were inversely related to blood pressure, formate (a byproduct of fermentation of dietary fibre by the gut microbiome), and hippurate (formed by hepatic glycine 
conjugation of benzoate, derived from gut microbial fermentation of plant phenolics). Alanine, on the other hand, which is higher in people who predominantly consume animal rather than vegetable products, was directly associated with blood pressure. Hippurate and 2-hydroxy-isobutyrate, derived from microbial degradation of dietary proteins, also discriminated between North and South Chinese populations, at differing cardiovascular risk (higher blood pressure and rates of stroke in North vs South China) [60].

The importance of the gut microbiome in cardiovascular phenotypes such as blood pressure is further supported by studies showing a link between trimethylamine- $N$-oxide (TMAO) and atherosclerosis [78]; TMAO, a small-molecule metabolite derived from the metabolism of dietary phosphatidylcholine, has been associated with risk of atherosclerosis and heart disease in observational studies and mouse models $[79,80]$. In relation to blood pressure, $\mathrm{Li}$ et al. found decreased microbial richness and diversity in prehypertensive and hypertensive populations compared with healthy controls [81•] while in rats, TMAO infusion was found to enhance the hypertensive effects of angiotensin II [82].

A role for the gut microbiome in high blood pressure was also reported in analysis of data from 896 normotensive black participants in the Atherosclerosis Risk in Communities (ARIC) study [57]. Serum samples were analysed by GSMS using the Metabolon platform; 4-hydroxyhippurate, an end product of benzoate metabolism from microbial fermentation of polyphenols, was associated with $17 \%$ higher risk of hypertension at 10 years follow-up, after adjusting for baseline blood pressure and traditional risk factors (hazard ratio per SD (95\%CI) $1.17(1.08,1.28))$. In addition, a sex steroid pattern derived from principal components analysis (PCA) was positively associated with elevated risk of incident hypertension (highest versus lowest quintile of the sex steroid scores, HR 1.72).

In the European Prospective Investigation into Cancer and Nutrition (EPIC)-Postdam study [68・•], 127 metabolites were analysed among 135 cases (participants who developed hypertension over 10 years follow-up) and 981 non-cases, using a targeted MS platform in blood samples (Biocrates); serine, glycine, acyl-alcyl-phospatidylcholines (PCs), and diacyl-PCs were associated with incident hypertension. These results suggest a possible role of inflammatory pathways in high blood pressure; both serine and glycine share anti-inflammatory and antioxidant properties, while PCs may also exhibit an anti-inflammatory role and protect lipoproteins from oxidation. Other cross-sectional studies with targeted metabolic profiling have supported the role of inflammation and oxidative stress in high blood pressure $[65,70]$.

Finally, a number of studies have supported the associations between lipid $[63 \bullet \cdot, 66,83]$ and amino acid $[62,64$, $68 \bullet^{\circ}$ ] metabolism with blood pressure. The TWINUK study with measurement of 280 metabolites in fasting serum samples (MS-based metabolic profiling using Metabolon platform) on 3580 females with replication in two independent cohorts: Cooperative Health Research in the Augsburg Region (KORA) $(n=1494)$ and Hertfordshire $(n=1515)$ [63.0. showed direct associations between hexadecanedioate (dicarboxylic acid) and both blood pressure and all-cause mortality. A causal role of this metabolite on blood pressure was supported by in vivo studies in rats highlighting the potential role of fatty acid $\omega$-oxidation in blood pressure regulation. Other smaller studies also suggested several lipids and amino acids associated with blood pressure levels [67, 69].

\section{Metabolic Phenotyping, Dietary Intervention Studies, and Blood Pressure}

The response of blood pressure regulation to dietary interventions has been investigated through metabolic phenotyping approaches in several studies, where different dietary patterns were adopted including the DASH Diet [50-52], Optimal Macronutrient Intake Trial for Heart Health (OmniHeart) Diets [56••], and Mediterranean Diet [61].

The metabolic response to OmniHeart diet [56••] was studied by ${ }^{1} \mathrm{H}$ NMR of stored 24-h urine samples among 58 individuals with prehypertension or stage 1 hypertension. In a randomised crossover design, participants received a carbohydrate rich (OMniCarbo), a protein rich (OmniProt), or a monounsaturated fat-rich (OmniMFA) diet, for 6 weeks each. Blood pressure was significantly associated with six urinary metabolites reflecting (i) dietary intake: proline-betaine (inverse) and carnitine (direct); (ii) gut microbial co-metabolites: hippurate (direct), 4-cresyl sulfate (inverse), and phenylacetylglutamine (inverse); and (iii) tryptophan metabolism: N-methyl-2pyridone-5-carboxamide (inverse). These results demonstrate, in a trial setting, changes in metabolic profiles from manipulation of dietary macronutrient content that lowers blood pressure levels.

Metabolic phenotyping in relation to dietary sodium reduction has also been studied. The response of dietary sodium reduction (DSR) was studied in a 10-week crossover RCT study among 17 adults with elevated systolic blood pressure (130-159 mmHg) [51]. Ten (of 289) measured urinary metabolites were significantly altered (nine upregulated and one downregulated) during low-sodium diet. These metabolites were involved in biologic pathways of nitric oxide production, oxidative stress, and osmotic regulation. Moreover, in the DASH-sodium randomised crossover trial [52], with individuals assigned to either DASH diet or control diet for 12 weeks, receiving in random order high, medium, and low amount of sodium for 30 days, 531 plasma metabolites were measured among 73 participants at the end of their high- and low-sodium interventions and among 46 participants at the end of their high- and 
medium-sodium interventions. 4-Ethylphenylsulfate, a xenobiotic produced by the gut microflora related to benzoate metabolism, increased with sodium reduction, suggesting that sodium intake may affect the gut microbial activity.

\section{Metabolic Phenotyping and Response to Therapy}

Several studies have attempted to identify potential biomarkers of drug responsiveness and to understand the molecular mechanisms that lead to drug response variation in blood pressure. For example, 313 Finnish men with high blood pressure (aged 35 to 60 years) from the Genetics of Drug Responsiveness in Essential Hypertension (GENRES) study received in a double-blind rotational design amlodipine, bisoprolol, hydrochlorothiazide (HCTZ), and losartan, each as monotherapy for 1 month, with 1-month placebo cycles between each treatment [71]. Treatment led to decreased long-chain acyl carnitines (amlodipine, bisoprolol, losartan) and medium and long-chain fatty acids (bisoprolol). Hexadecanedioate, a fatty acid previously linked to hypertension, was reduced after treatment with amlodipine, while HCTZ was, as expected, associated with increased uric acid. Although the study failed to identify a potential biomarker of drug response, it underscored the importance of fatty acids metabolism in hypertension. A lipidomics-based study [72] of 25 patients with essential hypertension on antihypertensive drug therapy, compared with 30 untreated patients with essential hypertension and 28 normotensive participants, also provided evidence supporting perturbed plasma lipid metabolism associated with hypertension. Specifically, triglycerides and total cholesteryl esters were significantly higher in hypertensive compared with normotensive participants, but significantly decreased in hypertensive patients after treatment.

\section{Conclusions}

A variety of metabolomic approaches applied to a range of epidemiological studies has been used to uncover the pathophysiology of high blood pressure. Several pathways have been suggested in relation to blood pressure including the

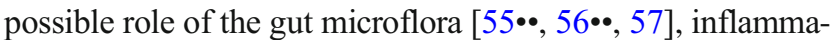
tory $[68 \bullet \bullet]$, oxidative stress $[65,70]$, and lipid pathways [63・•, $66,83]$. Metabolic changes have also been identified associated with blood pressure lowering effects of diets high in fruits and vegetables and low in meat intake $[51,52,56 \bullet \bullet]$. However, the current body of literature on metabolic profiling and blood pressure is still in its infancy, not fully consistent and requires careful interpretation. The majority of studies are small cross-sectional investigations in which the temporality of the relationship between exposure (metabolite) and outcome (high blood pressure) is uncertain, limiting any causal interpretation. In addition, independent replication efforts are rare and are further impaired by metabolic profiling methodologies that are difficult to integrate and harmonise between studies. To that end, metabolomics research should adapt practices such as those that are common in genetic epidemiology including correction for multiple testing and independent replications of findings in other populations. Similarly, functional follow-up studies that investigate the role of the metabolites in a physiological pathway such as animal models and human cell lines are necessary to further elucidate causal associations. Mendelian Randomisation approaches [84] are another approach to investigate the causality and direction of associations although they may face challenges in metabolomics research due to the large inter-correlations between metabolites and widespread pleiotropy. [85] Finally, integration of metabolomics with other omic methods is a promising approach to uncover the complex pathophysiology of hypertension by throwing light onto metabolic pathways linking, for example, genetic [86 $6^{\bullet}$ or epigenetic loci to high blood pressure.

Sources of Funding The work presented was carried out within the framework of a Stavros Niarchos Foundation grant to Aikaterini Iliou at the National and Kapodistrian University of Athens. Dr. Elliott is Director of the Medical Research Council-Public Health England Center for Environment and Health and acknowledges support from the Medical Research Council and Public Health England (MR/L01341X/1). He also acknowledges support from the National Institute of Health Research Biomedical Research Center at Imperial College London, and the National Institute of Health Research Health Protection Research Unit in Health Impact of Environmental Hazards (HPRU-2012-10141). Dr. Elliott is a UK Dementia Research Institute Professor, UK Dementia Research Institute at Imperial College London. This work was supported by the UK Dementia Research Institute which receives its funding from UK Dementia Research Institute Ltd. funded by the UK Medical Research Council, Alzheimer's Society and Alzheimer's Research UK. Dr. Elliott is associate director of the Health Data Research (HDR-UK) London which receives its funding from a consortium led by the UK Medical Research Council. This work used the computing resources of the UK MEDical BIOinformatics partnership (UK MED-BIO) which is supported by the Medical Research Council (MR/L01632X/1).

\section{Compliance with Ethical Standards}

Conflict of Interest The authors declare no conflicts of interest relevant to this manuscript.

Human and Animal Rights and Informed Consent This article does not contain any studies with human or animal subjects performed by any of the authors. 
Open Access This article is distributed under the terms of the Creative Commons Attribution 4.0 International License (http:// creativecommons.org/licenses/by/4.0/), which permits unrestricted use, distribution, and reproduction in any medium, provided you give appropriate credit to the original author(s) and the source, provide a link to the Creative Commons license, and indicate if changes were made.

\section{References}

Papers of particular interest, published recently, have been highlighted as:

- Of importance

•- Of major Importance

1. Tzoulaki I, Elliott P, Kontis V, Ezzati M. Worldwide exposures to cardiovascular risk factors and associated health effects: current knowledge and data gaps. Circulation. 2016;133:2314-33. This useful review describes the association between blood pressure and cardiovascular diseases and potential mechanisms.

2. Lewington S, Clarke R, Qizilbash N, Peto R, Collins R. Prospective studies collaboration. Age-specific relevance of usual blood pressure to vascular mortality: a meta-analysis of individual data for one million adults in 61 prospective studies. Lancet Lond Engl. 2002;360:1903-13.

3. Appel LJ, Brands MW, Daniels SR, Karanja N, Elmer PJ, Sacks FM, et al. Dietary approaches to prevent and treat hypertension: a scientific statement from the American Heart Association. Hypertens Dallas Tex 1979. 2006;47:296-308.

4. Whelton PK, He J, Appel LJ, Cutler JA, Havas S, Kotchen TA, et al. Primary prevention of hypertension: clinical and public health advisory from the National High Blood Pressure Education Program. JAMA. 2002;288:1882-8.

5. Elliott P, Stamler J, Nichols R, Dyer AR, Stamler R, Kesteloot H, et al. Intersalt revisited: further analyses of 24 hour sodium excretion and blood pressure within and across populations. Intersalt Cooperative Research Group BMJ. 1996;312:1249-53.

6. Xin X, He J, Frontini MG, Ogden LG, Motsamai OI, Whelton PK. Effects of alcohol reduction on blood pressure: a meta-analysis of randomized controlled trials. Hypertens Dallas Tex 1979. 2001;38: 1112-7.

7. Appel LJ, Moore TJ, Obarzanek E, Vollmer WM, Svetkey LP, Sacks FM, et al. A clinical trial of the effects of dietary patterns on blood pressure. DASH Collaborative Research Group N Engl J Med. 1997;336:1117-24.

8. Appel LJ, Sacks FM, Carey VJ, Obarzanek E, Swain JF, Miller ER, et al. Effects of protein, monounsaturated fat, and carbohydrate intake on blood pressure and serum lipids: results of the OmniHeart randomized trial. JAMA. 2005;294:2455-64.

9.• Tzoulaki I, Ebbels TMD, Valdes A, Elliott P, Ioannidis JPA. Design and analysis of metabolomics studies in epidemiologic research: a primer on-omic technologies. Am J Epidemiol. 2014;180:129-39. A useful tutorial on the reporting and analyses of metabolomics in epidemiological research.

10. Nicholson JK, Wilson ID. Understanding "global" systems biology: metabonomics and the continuum of metabolism. Nat Rev Drug Discov. 2003;2:668-76.

11. Bictash M, Ebbels TM, Chan Q, Loo RL, Yap IKS, Brown IJ, et al. Opening up the "black box": metabolic phenotyping and metabolome-wide association studies in epidemiology. J Clin Epidemiol. 2010;63:970-9.
12. Nicholson JK, Holmes E, Kinross JM, Darzi AW, Takats Z, Lindon JC. Metabolic phenotyping in clinical and surgical environments. Nature. 2012;491:384-92. A state of the art review on applications of metabolic phenotyping in biomedical research and clinical practice.

13. Holmes E, Wilson ID, Nicholson JK. Metabolic phenotyping in health and disease. Cell. 2008;134:714-7.

14. Bingol K, Brüschweiler R. Two elephants in the room: new hybrid nuclear magnetic resonance and mass spectrometry approaches for metabolomics. Curr Opin Clin Nutr Metab Care. $2015 ; 18: 471-7$.

15. Gartland KP, Beddell CR, Lindon JC, Nicholson JK. Application of pattern recognition methods to the analysis and classification of toxicological data derived from proton nuclear magnetic resonance spectroscopy of urine. Mol Pharmacol. 1991;39:629-42.

16. Lindon JC, Holmes FE, Nicholson JK. Pattern recognition methods and applications in biomedical magnetic resonance. Prog Nucl Magn Reson Spectrosc [Internet]. 2001 [cited 2018 May 16]; Available from: https://www.scienceopen.com/document?vid= 51e2f31b-895b-4031-9b45-36be03d9ee6d

17. Holmes E, Nicholson JK, Tranter G. Metabonomic characterization of genetic variations in toxicological and metabolic responses using probabilistic neural networks. Chem Res Toxicol. 2001;14:182-91.

18. Cloarec O, Dumas M-E, Craig A, Barton RH, Trygg J, Hudson J, et al. Statistical total correlation spectroscopy: an exploratory approach for latent biomarker identification from metabolic $1 \mathrm{H}$ NMR data sets. Anal Chem. 2005;77:1282-9.

19. Fan TW-M, Lane AN. Applications of NMR spectroscopy to systems biochemistry. Prog Nucl Magn Reson Spectrosc. 2016;92-93: $18-53$.

20. Nagana Gowda GA, Raftery D. Can NMR solve some significant challenges in metabolomics? J Magn Reson San Diego Calif 1997. 2015;260:144-60.

21. Dumas M-E, Maibaum EC, Teague C, Ueshima H, Zhou B, Lindon JC, et al. Assessment of analytical reproducibility of $1 \mathrm{H} \mathrm{NMR}$ spectroscopy based metabonomics for large-scale epidemiological research: the INTERMAP study. Anal Chem. 2006;78:2199-208.

22. Chen J, Singer S. High-resolution magic angle spinning NMR spectroscopy. Handb Metabonomics Metabolomics 2007. p. 113-47.

23. Zhang B, Xie M, Bruschweiler-Li L, Brüschweiler R. Nanoparticleassisted removal of protein in human serum for metabolomics studies. Anal Chem. 2016;88:1003-7.

24. Sellick CA, Hansen R, Stephens GM, Goodacre R, Dickson AJ. Metabolite extraction from suspension-cultured mammalian cells for global metabolite profiling. Nat Protoc. 2011;6:1241-9.

25. Huang Y, Zhang Z, Chen H, Feng J, Cai S, Chen Z. A highresolution $2 \mathrm{D} J$-resolved NMR detection technique for metabolite analyses of biological samples. Sci Rep. 2015;5:8390.

26. Silva Elipe MV. Advantages and disadvantages of nuclear magnetic resonance spectroscopy as a hyphenated technique. Anal Chim Acta. 2003;497:1-25.

27. Patti GJ, Yanes O, Siuzdak G. Metabolomics: the apogee of the omic triology. Nat Rev Mol Cell Biol. 2012;13:263-9.

28. Mahieu NG, Patti GJ. Systems-level annotation of a metabolomics data set reduces 25000 features to fewer than 1000 unique metabolites. Anal Chem. 2017;89:10397-406.

29. Xiao JF, Zhou B, Ressom HW. Metabolite identification and quantitation in LC-MS/MS-based metabolomics. TrAC Trends Anal Chem. 2012;32:1-14.

30. Lei Z, Huhman DV, Sumner LW. Mass spectrometry strategies in metabolomics. J Biol Chem. 2011;286:25435-42.

31. Ganna A, Fall T, Salihovic S, Lee W, Broeckling CD, Kumar J, et al. Large-scale non-targeted metabolomic profiling in three human population-based studies. Metabolomics. 2016;12(4) An example of large-scale metabolic phenotyping in epidemiological studies. 
32. Dunn WB, Broadhurst D, Begley P, Zelena E, FrancisMcIntyre S, Anderson N, et al. Procedures for large-scale metabolic profiling of serum and plasma using gas chromatography and liquid chromatography coupled to mass spectrometry. Nat Protoc. 2011;6:1060-83.

33. Begou O, Gika HG, Wilson ID, Theodoridis G. Hyphenated MSbased targeted approaches in metabolomics. Analyst. 2017;142: 3079-100.

34. Gorrochategui E, Jaumot J, Lacorte S, Tauler R. Data analysis strategies for targeted and untargeted LC-MS metabolomic studies: overview and workflow. TrAC Trends Anal Chem. 2016;82:425-42.

35. Schrimpe-Rutledge AC, Codreanu SG, Sherrod SD, McLean JA. Untargeted metabolomics strategies-challenges and emerging directions. J Am Soc Mass Spectrom. 2016;27:1897-905.

36. Nicholson JK, Foxall PJD, Spraul M, Farrant RD, Lindon JC. 750 $\mathrm{MHz} 1 \mathrm{H}$ and 1H-13C NMR spectroscopy of human blood plasma. Anal Chem. 1995;67:793-811.

37. Keun HC, Beckonert O, Griffin JL, Richter C, Moskau D, Lindon JC, et al. Cryogenic probe 13C NMR spectroscopy of urine for metabonomic studies. Anal Chem. 2002;74:4588-93.

38. Lamers R-JAN, Wessels ECHH, van de Sandt JJM, Venema K, Schaafsma G, van der Greef J, et al. A pilot study to investigate effects of inulin on Caco-2 cells through in vitro metabolic fingerprinting. J Nutr. 2003;133:3080-4.

39. Villas-Bôas SG, Højer-Pedersen J, Akesson M, Smedsgaard J, Nielsen J. Global metabolite analysis of yeast: evaluation of sample preparation methods. Yeast Chichester Engl. 2005;22:1155-69.

40. Price KE, Vandaveer SS, Lunte CE, Larive CK. Tissue targeted metabonomics: metabolic profiling by microdialysis sampling and microcoil NMR. J Pharm Biomed Anal. 2005;38:904-9.

41. Tomlins AM, Foxall PJD, Lindon JC, Nicholson JK, Lynch MJ, Spraul M, et al. High resolution magic angle spinning $1 \mathrm{H}$ nuclear magnetic resonance analysis of intact prostatic hyperplastic and tumour tissues. Anal Commun. 1998;35:113-5.

42. Karaman I. Preprocessing and Pretreatment of metabolomics data for statistical analysis. Adv Exp Med Biol. 2017:145-61.

43. Rusilowicz M, Dickinson M, Charlton A, O'Keefe S, Wilson J. A batch correction method for liquid chromatography-mass spectrometry data that does not depend on quality control samples. Metabolomics [Internet]. 2016;12. Available from: https://www. ncbi.nlm.nih.gov/pmc/articles/PMC4757603/

44. Savorani F, Tomasi G, Engelsen SB. icoshift: a versatile tool for the rapid alignment of 1D NMR spectra. J Magn Reson. 2010;202: 190-202.

45. Veselkov KA, Lindon JC, Ebbels TMD, Crockford D, Volynkin VV, Holmes E, et al. Recursive segment-wise peak alignment of biological (1)h NMR spectra for improved metabolic biomarker recovery. Anal Chem. 2009;81:56-66.

46. Pearce JTM, Athersuch TJ, Ebbels TMD, Lindon JC, Nicholson JK, Keun HC. Robust algorithms for automated chemical shift calibration of 1D 1H NMR spectra of blood serum. Anal Chem. 2008;80:7158-62.

47. Dieterle F, Ross A, Schlotterbeck G, Senn H. Probabilistic quotient normalization as robust method to account for dilution of complex biological mixtures. Application in 1H NMR metabonomics. Anal Chem. 2006;78:4281-90.

48. Karaman I, Ferreira DLS, Boulangé CL, Kaluarachchi MR, Herrington D, Dona AC, et al. Workflow for integrated processing of multicohort untargeted 1H NMR metabolomics data in largescale metabolic epidemiology. J Proteome Res. 2016;15:4188-94. An example of NMR preprocessing using metabolic phenotyping data from different epidemiological studies.

49. Fages A, Pontoizeau C, Jobard E, Lévy P, Bartosch B, ElenaHerrmann B. Batch profiling calibration for robust NMR metabonomic data analysis. Anal Bioanal Chem. 2013;405: 8819-27.

50. Mathew AV, Seymour EM, Byun J, Pennathur S, Hummel SL. Altered metabolic profile with sodium-restricted dietary approaches to stop hypertension diet in hypertensive heart failure with preserved ejection fraction. J Card Fail. 2015;21:963-7.

51. Jablonski KL, Klawitter J, Chonchol M, Bassett CJ, Racine ML, Seals DR. Effect of dietary sodium restriction on human urinary metabolomic profiles. Clin J Am Soc Nephrol CJASN. 2015;10: 1227-34.

52. Derkach A, Sampson J, Joseph J, Playdon MC, StolzenbergSolomon RZ. Effects of dietary sodium on metabolites: the dietary approaches to stop hypertension (DASH)-sodium feeding study. Am J Clin Nutr. 2017;106:1131-41.

53. van Deventer CA, Lindeque JZ, van Rensburg PJJ, Malan L, van der Westhuizen FH, Louw R. Use of metabolomics to elucidate the metabolic perturbation associated with hypertension in a black South African male cohort: the SABPA study. J Am Soc Hypertens. 2015;9:104-14.

54. Kang YM, Jung CH, Jang JE, Hwang JY, Kim EH, Park J-Y, et al. The association of incident hypertension with metabolic health and obesity status: definition of metabolic health does not matter. Clin Endocrinol. 2016;85:207-15.

55.• Holmes E, Loo RL, Stamler J, Bictash M, Yap IKS, Chan Q, et al. Human metabolic phenotype diversity and its association with diet and blood pressure. Nature. 2008;453:396-400. This landmark study, using high-quality epidemiological data, investigated large-scale urine metabolic phenotyping with blood pressure across 17 population samples.

$56 . \bullet$ Loo RL, Zou X, Appel LJ, Nicholson JK, Holmes E. Characterization of metabolic responses to healthy diets and association with blood pressure: application to the Optimal Macronutrient Intake Trial for Heart Health (OmniHeart), a randomized controlled study. Am J Clin Nutr. 2018;107:323-34. This recent study represents one of the largest dietary interventions using a metabolic profiling approach to investigate the metabolic responses to healthy diets, and subsequently to define the association of these metabolites with improvement of risk factors for cardiovascular diseases.

57. Zheng Y, Yu B, Alexander D, Mosley TH, Heiss G, Nettleton JA, et al. Metabolomics and incident hypertension among blacks: the atherosclerosis risk in communities study. Hypertens Dallas Tex 1979. 2013;62:398-403.

58. Jennings A, MacGregor A, Pallister T, Spector T, Cassidy A. Associations between branched chain amino acid intake and biomarkers of adiposity and cardiometabolic health independent of genetic factors: a twin study. Int J Cardiol. 2016;223:992-8.

59. Mozaffarian D, de Oliveira Otto MC, Lemaitre RN, Fretts AM, Hotamisligil G, Tsai MY, et al. Trans-palmitoleic acid, other dairy fat biomarkers, and incident diabetes: the multi-ethnic study of atherosclerosis (MESA). Am J Clin Nutr. 2013;97:854-61.

60. Yap IKS, Brown IJ, Chan Q, Wijeyesekera A, Garcia-Perez I, Bictash M, et al. Metabolome-wide association study identifies multiple biomarkers that discriminate north and south Chinese populations at differing risks of cardiovascular disease: INTERMAP study. J Proteome Res. 2010;9:6647-54.

61. Sleiman D, Al-Badri MR, Azar ST. Effect of mediterranean diet in diabetes control and cardiovascular risk modification: a systematic review. Front Public Health. 2015;3:69.

62. Ameta K, Gupta A, Kumar S, Sethi R, Kumar D, Mahdi AA. Essential hypertension: a filtered serum based metabolomics study. Sci Rep. 2017;7:2153.

63.• Menni C, Graham D, Kastenmüller G, Alharbi NHJ, Alsanosi SM, McBride M, et al. Metabolomic identification of a novel pathway of blood pressure regulation involving hexadecanedioate novelty and significance. Hypertension. 2015;66:422-9. This study revealed 
that hexadecanedioate was associated with blood pressure regulation with replication of findings in other populations and independent animal experiments to support causality.

64. Zhong L, Zhang J-P, Nuermaimaiti A-G, Yunusi K-X. Study on plasmatic metabolomics of Uygur patients with essential hypertension based on nuclear magnetic resonance technique. Eur Rev Med Pharmacol Sci. 2014;18:3673-80.

65. Kim M, Jung S, Kim SY, Lee S-H, Lee JH. Prehypertensionassociated elevation in circulating lysophosphatidlycholines, LpPLA2 activity, and oxidative stress. PLoS One. 2014;9:e96735.

66. Kulkarni H, Meikle PJ, Mamtani M, Weir JM, Barlow CK, Jowett $\mathrm{JB}$, et al. Plasma lipidomic profile signature of hypertension in Mexican American families: specific role of diacylglycerols. Hypertens Dallas Tex 1979. 2013;62:621-6.

67. Yang M, Yu Z, Deng S, Chen X, Chen L, Guo Z, et al. A targeted metabolomics MRM-MS study on identifying potential hypertension biomarkers in human plasma and evaluating acupuncture effects. Sci Rep. 2016;6:25871.

68.• Dietrich S, Floegel A, Weikert C, Prehn C, Adamski J, Pischon T, et al. Identification of serum metabolites associated with incident hypertension in the European Prospective Investigation into Cancer and Nutrition-Potsdam Study. Hypertension. 2016;68:471-7. This population-based prospective cohort study with long follow-up time revealed metabolic alterations that occur early in the development of hypertension, suggesting a possible role of inflammatory pathways in high blood pressure.

69. Wang L, Hou E, Wang L, Wang Y, Yang L, Zheng X, et al. Reconstruction and analysis of correlation networks based on GC-MS metabolomics data for young hypertensive men. Anal Chim Acta. 2015;854:95-105.

70. De Meyer T, Sinnaeve D, Van Gasse B, Tsiporkova E, Rietzschel ER, De Buyzere ML, et al. NMR-based characterization of metabolic alterations in hypertension using an adaptive, intelligent binning algorithm. Anal Chem. 2008;80:3783-90.

71. Hiltunen TP, Rimpelä JM, Mohney RP, Stirdivant SM, Kontula KK. Effects of four different antihypertensive drugs on plasma metabolomic profiles in patients with essential hypertension. PLoS One. 2017;12:e187729.

72. Hu C, Kong H, Qu F, Li Y, Yu Z, Gao P, et al. Application of plasma lipidomics in studying the response of patients with essential hypertension to antihypertensive drug therapy. Mol BioSyst. 2011;7: 3271-9.

73. Rotroff DM, Shahin MH, Gurley SB, Zhu H, Motsinger-Reif A, Meisner M, et al. Pharmacometabolomic assessments of atenolol and hydrochlorothiazide treatment reveal novel drug response phenotypes. CPT Pharmacomet Syst Pharmacol. 2015;4:669-79.

74. Wikoff WR, Frye RF, Zhu H, Gong Y, Boyle S, Churchill E, et al. Pharmacometabolomics reveals racial differences in response to atenolol treatment. PLoS One. 2013;8:e57639.
75. Altmaier E, Menni C, Heier M, Meisinger C, Thorand B, Quell J, et al. The pharmacogenetic footprint of ACE inhibition: a populationbased metabolomics study. PLoS One. 2016;11:e153163.

76. Shahin MH, Gong Y, McDonough CW, Rotroff DM, Beitelshees AL, Garrett TJ, et al. A genetic response score for hydrochlorothiazide use: insights from genomics and metabolomics integration. Hypertens Dallas Tex 1979. 2016;68:621-9.

77. Tomaszewski M, White C, Patel P, Masca N, Damani R, Hepworth $\mathrm{J}$, et al. High rates of non-adherence to antihypertensive treatment revealed by high-performance liquid chromatography-tandem mass spectrometry (HP LC-MS/MS) urine analysis. Heart Br Card Soc. 2014;100:855-61.

78. Wilson Tang WH, Hazen SL. The gut microbiome and its role in cardiovascular diseases. Circulation. 2017;135:1008-10.

79. Wang Z, Klipfell E, Bennett BJ, Koeth R, Levison BS, DuGar B, et al. Gut flora metabolism of phosphatidylcholine promotes cardiovascular disease. Nature. 2011;472:57-63.

80. Tang WHW, Wang Z, Levison BS, Koeth RA, Britt EB, Fu X, et al. Intestinal microbial metabolism of phosphatidylcholine and cardiovascular risk. N Engl J Med. 2013;368:1575-84.

81. Li J, Zhao F, Wang Y, Chen J, Tao J, Tian G, et al. Gut microbiota dysbiosis contributes to the development of hypertension. Microbiome. 2017;5(14). This study supported a causal role of aberrant gut microbiota contributing to the pathogenesis of hypertension).

82. Ufnal M, Jazwiec R, Dadlez M, Drapala A, Sikora M, Skrzypecki J. Trimethylamine-N-oxide: a carnitine-derived metabolite that prolongs the hypertensive effect of angiotensin II in rats. Can J Cardiol. 2014;30:1700-5.

83. Brindle JT, Nicholson JK, Schofield PM, Grainger DJ, Holmes E. Application of chemometrics to $1 \mathrm{H}$ NMR spectroscopic data to investigate a relationship between human serum metabolic profiles and hypertension. Analyst. 2003;128:32-6.

84. Boef AGC, Dekkers OM, le Cessie S. Mendelian randomization studies: a review of the approaches used and the quality of reporting. Int J Epidemiol. 2015;44:496-511.

85. Hemani G, Bowden J, Davey Smith G. Evaluating the potential role of pleiotropy in Mendelian randomization studies. Hum Mol Genet. 2018

86. Warren HR, Evangelou E, Cabrera CP, Gao H, Ren M, Mifsud B, et al. Genome-wide association analysis identifies novel blood pressure loci and offers biological insights into cardiovascular risk. Nat Genet. 2017;49:403-15. This large genome-wide association study revealed associations between genetic loci affecting blood pressure and circulating metabolites through metabolic phenotyping. 